\title{
DROGAS IMUNOSSUPRESSORAS AFETAM OS NÚCLEOS HIPOTALÂMICOS ENVOLVIDOS NA INGESTÃO ALIMENTAR ? ESTUDO EXPERIMENTAL
}

\author{
DO THE IMMUNOSUPPRESSIVE DRUGS AFFECT THE HYPOTHALAMIC NUCLEI INVOLVED IN THE REGULATION OF \\ FOOD INTAKE? AN EXPERIMENTAL STUDY
}
Rodrigo SCHUH ${ }^{1,2}{ }^{\odot}$, Djanira Aparecida da Luz VERONEZ ${ }^{2}{ }^{\circ}$, Eduardo José Brommelstroet RAMOS ${ }^{2}$, Flávia Dorieux Wastner CUNHA $^{2 \oplus}{ }^{\circ}$, Mattheus Lopes PEREIRA ${ }^{2 \oplus}$, Jeferson de Jesus ARANHA ${ }^{2 \odot}$, Marcelo Alves ARANHA ${ }^{10}$, Jorge Eduardo Fouto MATIAS ${ }^{1}$

\begin{abstract}
RESUMO - RACIONAL: Drogas imunossupressoras são indispensáveis para pacientes pós-transplante diminuindo, significativamente, os riscos de rejeição inerentes a este tipo de procedimento. No entanto, seus efeitos colaterais sobre os núcleos hipotalâmicos envolvidos na regulação da ingestão de alimentos e o efeito no excessivo ganho de peso e suas comorbidades associadas são desconhecidos. OBJETIVO: Analisar a ocorrência de alterações morfológicas dos núcleos paraventricular, área hipotalâmica lateral, dorsomedial, ventromedial e arqueado em ratos Wistar submetidos ao tratamento imunossupressor com Tacrolimus (TAC) ou Micofenolato Mofetil (MMF). MÉTODOS: Foram utilizados Ratos Wistar machos adultos distribuídos, randomicamente, em quatro grupos de acordo com o tratamento oral utilizado por 14 semanas: Controle; Sham (Placebo); Tacrolimus (TAC $1 \mathrm{mg} / \mathrm{kg}$ peso) e Micofenolato Mofetil (MMF $30 \mathrm{mg} / \mathrm{kg}$ peso). Ao final do tratamento, os animais foram eutanasiados e seus encéfalos fixados para o processamento histológico. Posteriormente, as lâminas foram fotodocumentadas para o desenvolvimento da análise estereológica dos corpos celulares dos neurônios dos núcleos hipotalâmicos, tendo como parâmetros a densidade neuronal e no número de neurônios. RESULTADOS: Todos os grupos estudados mostraram curva de ganho de peso ponderal durante todo o período de experimento. Não houve diferença significativa na densidade neuronal e no número de neurônios hipotalâmicos dos núcleos hipotalâmicos entre os grupos estudados. Não foram detectadas alterações morfológicas dos corpos celulares dos neurônios hipotalâmicos capazes de serem imputadas ao uso dos imunossupressores envolvidos no estudo. CONCLUSÃO O tratamento dos animais experimentais com os imunossupressores não evidenciou alterações no número e densidade dos corpos celulares dos neurônios dos núcleos hipotalâmicos estudados.

DESCRITORES: Ácido Micofenólico. Hipotálamo. Obesidade. Tacrolimo. Transplantes
\end{abstract}

ABSTRACT - BACKGROUND: Immunosuppressive drugs are essential for reducing the rejection risk in post-transplant patients, which is commonly associated with this procedure. However, side effects of those drugs on the hypothalamic nuclei involved in the food intake regulation, excessive weight gain, and also associated comorbidities are still unknown. PURPOSE: The purpose of this study was to analyze possible changes in the neuronal morphology and cell density in the paraventricular nuclei, lateral hypothalamic area, dorsomedial nuclei, and ventromedial and arcuate nuclei in Wistar rats submitted to immunosuppressive treatment with tacrolimus (TAC) or mycophenolate mofetil (MMF). METHODS: Adult male Wistar rats were randomly assigned to the following groups according to the oral treatment administered for 14 weeks: control, sham (placebo), TAC (1 mg/kg of weight), and MMF (30 mg/kg of weight). After treatment, the animals were sacrificed and their brains fixed for later histological staining. Subsequently, the slides were photodocumented for stereological analysis of the hypothalamic nuclei. RESULTS: All experimental groups showed a weight gain throughout the study. There was no significant difference in neuronal density/number of cells in the hypothalamic nuclei between groups. Morphological changes were not detected in the hypothalamic neurons. CONCLUSION: Treatments with immunosuppressants could not modify the morphological and cell density aspects of the hypothalamic nuclei during this supplementation period.

HEADINGS: Mycophenolic acid. Hypothalamus. Obesity. Tacrolimus. Transplants

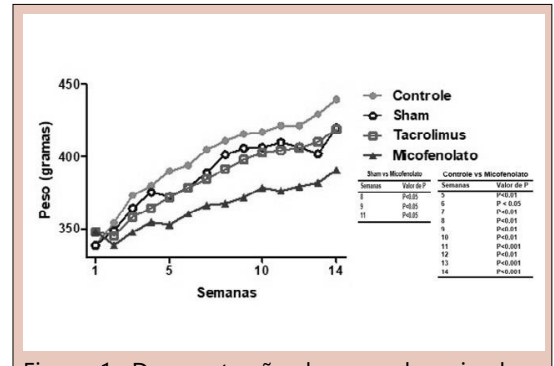

Figura 1- Demonstração do peso do animal ao longo de 14 semanas de tratamento ${ }^{*} p=0,05$. ANOVA de duas vias. $\mathrm{N}=6 /$ grupo.

\begin{tabular}{l}
\hline Mensagem central \\
\hline Há evidências de ganho de peso associado \\
a terapia imunossupressora pós-transplante \\
em humanos. A hipótese é que alterações \\
hipotalâmicas podem ocorrer como efeito \\
colateral da terapia imunossupressora com \\
Tacrolimus e Micofenolato de Mofetil, causando \\
ganho de peso e obesidade.
\end{tabular}

\section{Perspectivas}

Os tratamentos com imunossupressores TAC e MMF isolados não foram capazes de modificar os aspectos morfológicos e a densidade celular dos núcleos hipotalâmicos durante o período de suplementação. Não foi observada obesidade com o protocolo de administração de imunossupressor utilizado.

Trabalho realizado na 'Universidade Federal do Paraná - Departamento de Cirurgia - Curitiba - Paraná - Brasil, ${ }^{2}$ Universidade Federal do Paraná - Departamento de Anatomia - Curitiba - Paraná - Brasil

Como citar esse artigo:. Schuh R, Veronez DAL, Ramos EJB, Cunha FDW, Pereira ML, Aranha JJ, Aranha MA, Matias JEF. Drogas imunossupressoras afetam os núcleos hipotalâmicos envolvidos na ingestão alimentar? estudo experimental. ABCD Arq Bras Cir Dig. 2021;34(4):e1636. https://doi.org/10.1590/0102-672020210002e1636

Correspondência:

Rodrigo Schuh.

Email: rodrigoanato@gmail.com;

rodrigoanato@hotmail.com
Fonte de financiamento: Não

Conflito de interesses: Não

Recebido: 17/08/2021

Aceito: 17/10/2021 


\section{INTRODUÇÃO}

Nas últimas duas décadas, as taxas de sobrevida após o transplante de fígado aumentaram para $85 \%$ em cinco anos e para $56 \%$ após 20 anos, principalmente devido aos avanços nas técnicas cirúrgicas, manejo imunológico e cuidados pré e pós-operatórios ${ }^{5}$. No entanto, o aumento da sobrevida dos pacientes submetidos ao transplante hepático foi acompanhado por um aumento na prevalência de doenças crônicas, geralmente superior à prevalência encontrada na população em geral. Além disso, destaca-se o ganho de peso vivenciado por esses pacientes, gerando sobrepeso e obesidade com impacto na sobrevida ${ }^{25}$.

Da mesma forma, há relatos de aumento da obesidade em $40 \%$ dos pacientes no primeiro ano pós-transplante e, após três anos da cirurgia, cerca de $70 \%$ deles apresentavam excesso de peso corporal ${ }^{22}$.

Além disso, o ganho de peso após o transplante hepático pode ter diversos fatores como balanço energético positivo ${ }^{21}$, estilo devida sedentário ${ }^{13}$, desenvolvimento de estado hipometabólico ${ }^{23}$ e uso de terapias imunossupressoras ${ }^{7}$.

Portanto, há evidências de expressivo ganho de peso associado à terapia imunossupressora pós-transplante em humanos $1,4,13,14$

Desse modo, a principal hipótese é de que possíveis alterações hipotalâmicas podem ocorrer como efeito colateral da terapia imunossupressora com tacrolimus (TAC) e micofenolato mofetil (MMF), causando ganho de peso e obesidade. Adicionalmente, intervenções terapêuticas capazes de interromper ou limitar o envolvimento do hipotálamo podem se tornar possíveis novas estratégias terapêuticas para a prevenção da obesidade no tratamento de imunossupressores pós-transplante.

Poucos estudos pré-clínicos estão investigando mecanismos relacionados a terapias de imunossupressão e obesidade pós-transplante. Assim, os fatores neurotóxicos das drogas imunossupressoras e seus efeitos sobre os núcleos hipotalâmicos envolvidos coma regulação da ingestão alimentar e do metabolismo energético precisam ser estudados. A esse respeito, o objetivo do estudo foi analisar possíveis alterações na morfologia neuronal e densidade celular nos núcleos paraventriculares (PA), área hipotalâmica lateral (LH), núcleos dorsomedial (DM), ventromedial (VM) e núcleos arqueados (ARC) em ratos Wistar submetidos a tratamento imunossupressor com TAC ou MMF.

\section{MÉTODOS}

\section{Animais e aprovação ética}

Este estudo foi aprovado pelo Comitê de Ética no Uso de Animais em Pesquisa Experimental da Universidade Federal do Paraná (CEUA no 752). Para esta pesquisa, foram utilizados vinte e quatro ratos Wistar machos (Rattus novergicus albinus, Ordem Rodentia, Classe Mammalia), com 70 dias de idade, pesando entre 300 e 350 gramas, e fornecidos pelo Biotério do Setor de Ciências Biológicas da UFPR.

\section{Design experimental}

Os animais foram alojados em gaiolas apropriadas (máximo de 5 animais / gaiola) com temperatura controlada $\left(26 \pm 1^{\circ} \mathrm{C}\right)$ e ciclo claro-escuro (12:12h), umidade relativa de $45 \%$ e acesso a água e ração ad libitum. Após o período de aclimatação, os ratos foram distribuídos aleatoriamente em quatro grupos ( $N$ $=6$ / grupo), de acordo com o tratamento imunossupressor proposto (uma vez ao dia durante 14 semanas):

- Grupo Controle-Nenhum medicamento foi administrado.

- Grupo Sham - Administração de placebo - Solução Salina (SS) 0,9\% via gavagem.
- Grupo Tacrolimus - Administração de TAC (1 mg / kg) diluído em SS 0,9\%, via gavagem.

- Grupo Micofenolato - Administração de MMF (30mg / kg) diluído em SS 0,9\%, via gavagem.

\section{Administração de medicamentos e coleta de amostras}

Os medicamentos TAC e MMF foram diluídos em 0,9\% SS imediatamente antes de seu uso. Para fins de padronização, as diluições foram realizadas sempre seguindo o mesmo protocolo: $9 \mathrm{mg}$ de TAC diluído em $7 \mathrm{ml}$ de SS 0,9\% (1,28 mg / ml); 500 mg de MMF (1 comprimido) diluído em $30 \mathrm{ml}$ de SS $0,9 \%(16,7 \mathrm{mg} / \mathrm{ml})$. Após a diluição, o volume oferecido aos animais foi semelhante entre os grupos, calculado de acordo com o peso corporal de cada animal.

Os animais receberam o tratamento diariamente de acordo com o grupo por 14 semanas consecutivas. Drogas e placebo foram administrados por gavagem. Os animais foram imobilizados, a cânula orogástrica acoplada a uma seringa de $1 \mathrm{ml}$ e introduzida delicadamente pela cavidade oral, atingindo o esôfago e estômago. Após a verificação da passagem da cânula para o trato digestivo, a solução foi injetada suavemente, evitando o refluxo da solução e o desconforto do animal. Ao final do período de administração de 14 semanas, os animais foram anestesiados e sedados com quetamina (100 mg / kg) e xilazina (5 mg / kg) por via intraperitoneal.

Após a anestesia, a decapitação foi realizada por guilhotina manual seguida de trepanação e retirada do cérebro, os quais foram fixados em solução de Alfac (formaldeído (37-40\%), ácido acético glacial e álcool etílico $80^{\circ}$ ) por 16 horas e realocados em recipientes contendo álcool $70^{\circ}$ onde permaneceram até o momento do acondicionamento em resina Paraplast ${ }^{\circledast}$.

\section{Lâminas Histológicas}

Para microtomia e coloração histológica, cortes alternados e uniformemente isotrópicos ( $\mathrm{N}=6$ / coordenada) do cérebro foram realizados, usando o micrótomo Gebrauchshinweisefur Minot-Mikrotom, modelo 1212 da empresa E. LeitzWetzlar para obter os cortes de $7 \mu \mathrm{m}$ de espessura.

Asáreas específicas do hipotálamo de rato foram selecionadas de acordo com a Coordenada Estereotáxica (Bregma $=-1,56$ $\mathrm{mm}$ e Bregma $=-2,40 \mathrm{~mm})^{19}$.

Para a análise histológica, foi utilizado a coloração de Nissl e o material preparado em lâmina histológica com Entellan ${ }^{\circledR}$.

\section{Fotodocumentação e Quantificação}

Todas as lâminas histológicas foram fotodocumentadas no Laboratório Multiusuários de Microscopia de Fluorescência Convencional e Confocal e as imagens obtidas usando a câmera CoolCube 1 - Metasystems conectada ao microscópio Axiolmager Z2 (Carl Zeiss, Jena, DE), equipado com Metafer 4 / VSlide software de captura automatizada (Metasystems, Altlussheim, DE) e observado no software VsViewer ${ }^{\circledR}$ (Metasystems, Altlussheim, DE).

Posteriormente, com o objetivo de selecionar e obter o local desejado para análise em cada corte, as imagens obtidas foram aumentadas 30 vezes no software VsViewer ${ }^{\circledR}$, com ampliação e precisão adequadas para ajuste das máscaras pela precisão da localização e das grades do dissector físico, destinado a delimitar a área de quantificação. Para esta captura, foi utilizado um Ultrabook Asus S550C conectado a um monitor de vídeo Hewlett Packard EliteDisplay E241i, no qual havia uma distinção entre as áreas hipotalâmicas nos hemisférios cerebrais, gerando imagens distintas para cada antímero. Além disso, foi utilizado o software Photoshop CS 6Extended ${ }^{\circledR}$ (Adobe) para criar as máscaras a partir das imagens do Guia Estereotáxico (Paxinos and 
Watson n.d.), com o objetivo de delimitar a área do núcleo de cada uma dessas imagens e seu antímero correspondente. Posteriormente, foi confeccionado o Dissector Físico com dimensões de 200 × $200 \mu \mathrm{m}$, específico para a quantificação celular de cada núcleo.

Para a quantificação estereológica dos corpos celulares dos neurônios dos núcleos hipotalâmicos (ARC, PA, DM, VM e LH) foi utilizado o Princípio do Dissector Físico. Este sistema consiste na aplicação de um quadro formado por uma linha proibida e outra permitida, delimitando uma área de 40.000 $\mu \mathrm{m} 2$, onde apenas os corpos celulares dos neurônios do plano superior que estão dentro do quadro ou tocando sua linha permitida são contados.

Os seguintes parâmetros estereológicos dos corpos celulares dos neurônios dos núcleos hipotalâmicos foram determinados: densidade numérica (ND) e densidade de área (DA).

A quantificação foi obtida manualmente com auxílio do software Image $J^{\circledR}$, no qual todos os neurônios foram selecionados e identificados no Dissector Físico, sempre a partir das linhas de quantificação e suas restrições.

Para obter os parâmetros tridimensionais dos núcleos hipotalâmicos (ARC, PA, DM, VM e LH) foram aplicadas as seguintes equações:

Densidade numérica (ND) de corpos celulares de neurônios:

Vol[dissector] $=$ t.TA
$\mathrm{ND}=\mathrm{SQ} /$ Vol[dissector $](1 / \mathrm{mm} 3)$

$\mathrm{t}=$ espessura do corte histológico; TA = Área de teste do plano superior; $\mathrm{SQ}$ = número de corpos celulares de neurônios)

Para obter a densidade por área (DA):

$$
\mathrm{DA}=\mathrm{N} / \mathrm{TA}(\mu \mathrm{m} 3)
$$

( $\mathrm{N}$ = número de perfis de células observados na área de teste (TA))

\section{Análise Estatística}

Para análise estatística, a distribuição normal dos dados foi avaliada pelo teste de Kolmogorov-Smirnov, e os dados foram considerados paramétricos. Para avaliação do peso dos animais, foi realizado o teste de análise de variância (ANOVA) de duas vias, seguido do pós-teste de Bonferroni. O teste t de Student foi realizado para avaliar possíveis diferenças entre os hemisférios cerebrais, nos núcleos avaliados. As diferenças entre os grupos, considerando os dois hemisférios das secções, foram avaliadas pelo teste de análise de variância (ANOVA) de uma via, seguido do pós-teste de Newman-Keuls. Os valores foram expressos como média \pm erro padrão da média e o nível de significância foi estabelecido em $p=0,05$.

O software utilizado para análise estatística e geração de gráficos foi o Prisma (GraphPad Prism ${ }^{\circledR}$, versão 5.01).
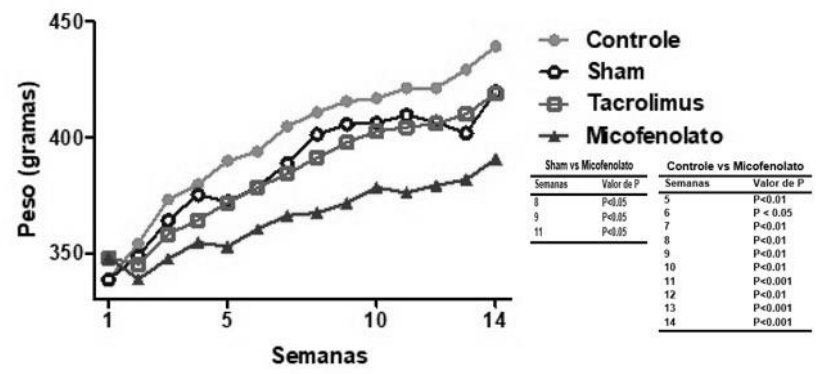

Figura 1 - Demonstração do peso do animal ao longo de 14 semanas de tratamento ${ }^{*} p=0,05$. ANOVA de duas vias. $\mathrm{N}=6 /$ grupo.
RESULTADOS

Todos os animais ganharam peso ao longo do estudo (Figura 1), porém, não foi possível identificar aumento significativo de peso nos animais tratados com imunossupressor TAC quando comparados aos grupos Controle e Sham. Analisando o grupo MMF, houve menor ganho de peso em comparação aos grupos Sham (8, 9 e 11 semanas) e Controle (5-14 semanas).

\section{Núcleo Paraventricular}

Comparando a densidade neuronal do Núcleo Paraventricular entre os grupos (Figura 2), observou-se que não houve diferença estatística entre eles $[F(3,20)=2,327 ; P=0,1054]$.

\section{Área Hipotalâmica Lateral}

A densidade neuronal da Área Hipotalâmica Lateral não apresentou variações significativas (Figura 3) quando comparada entre os grupos estudados $[F(3,20)=2,618 ; P=0,0792]$.

\section{Núcleo Dorsomedial}

A área do Núcleo Dorsomedial apresentou valores semelhantes (Figura 4) quanto à densidade neuronal analisada em cada grupo de estudo $[\mathrm{F}(3,20)=1,104 ; \mathrm{P}=0,3707]$.

\section{Núcleo Ventromedial}

Considerando a área do Núcleo Ventromedial, não foi detectada diferença estatística entre os grupos (Figura 5) quando calculada a densidade neuronal $[F(3,20)=1,641 ; P=0,2117]$.

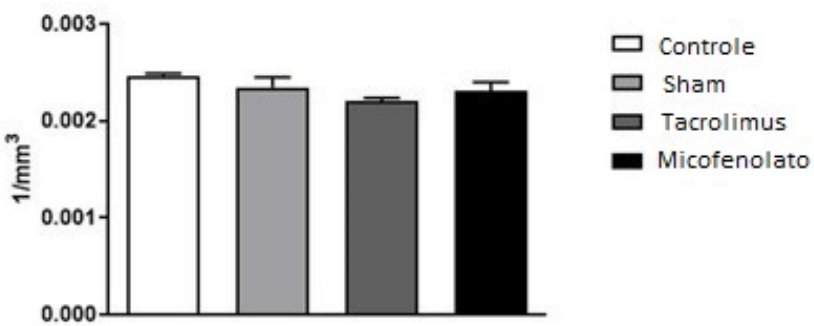

Figura 2 - Morfo-quantificação dos neurônios paraventriculares, ANOVA de uma via. Média \pm erro da média. $\mathrm{N}=$ 6 / grupo.

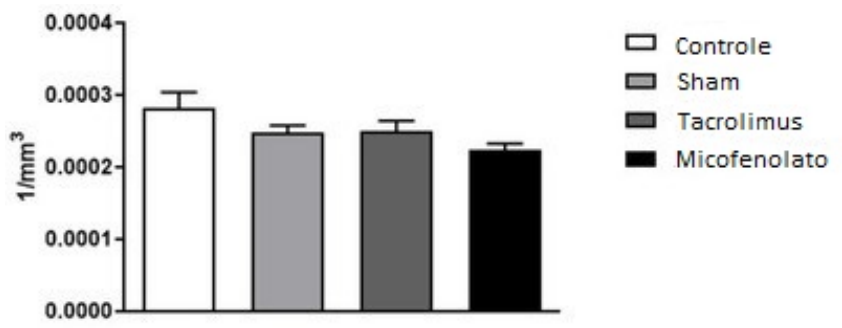

Figura 3 - Morfo-quantificação dos neurônios daárea hipotalâmica lateral. ANOVA de uma via. Média \pm erro da média. $\mathrm{N}=6 /$ grupo.

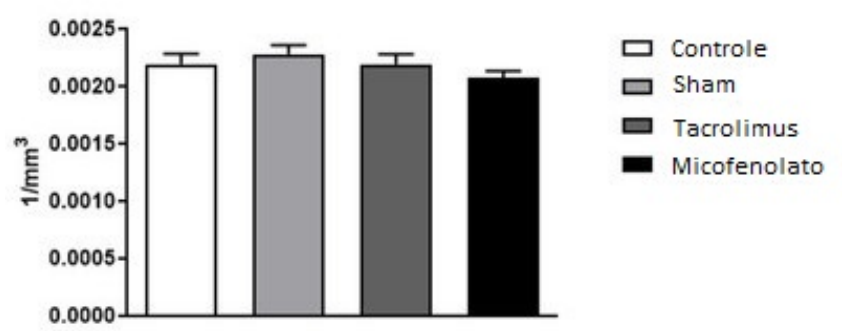

Figura 4 - Morfo-quantificação dos neurônios dorsomediais, ANOVA de uma via. Média \pm erro da média. $\mathrm{N}=$ $6 /$ grupo. 


\section{Núcleo Arqueado}

A área do Núcleo Arqueado também não apresentou diferença significativa (Figura 6) entre a densidade neuronal observada para cada grupo de estudo $[F(3,20)=2,133$; $P=0,1281]$.

\section{DISCUSSÃO}

O modelo experimental foi desenvolvido em consonância com os princípios gerais das Diretrizes Brasileiras de Cuidado e Uso de Animais para fins científicos e educacionais ${ }^{15}$, que incentiva a adesão e aplicação dos princípios dos $3 R$ s (traduzidos do inglês: substituir, reduzir e refinar). Os animais tratados com Tacrolimus e Micofenolato Mofetil para o modelo experimental de análise quantitativa de neurônios foram obtidos a partir do reaproveitamento de ratos Wistar albinos machos (Rattus norvegicus albinus, mamífero Rodentia) de pesquisa anterior: "Avaliação da espermatogênese em ratos imunossupressores", sob protocolo $n^{\circ} 752$, certificado pelo Comitê de Ética no Uso de Animais (CEUA), Setor de Ciências Biológicas da Universidade Federal do Paraná. Assim, para o desenvolvimento deste ensaio, fez-se o uso experimental de animais para quantificação de neurônios nos núcleos hipotalâmicos PA, LH, DM, VM e ARC de ratos Wistar machos tratados ou não com os imunossupressores MMF e TAC. Dessa forma, formou-se o Grupo Controle, sem administração de medicamentos; o grupo Tacrolimus com administração de dose diária de TAC (1 $\mathrm{mg} / \mathrm{kg}$ peso) diluído em solução fisiológica 0,9\%; o grupo Micofenolato com administração de dose diária de MMF (30 mg / kg peso) diluído em solução fisiológica 0,9\% e o grupo Sham com administração de solução fisiológica 0,9\%. O método de gavagem foi usado para administração de fármaco / veículo.

O método estereológico para estudo morfoquantitativo dos corpos celulares do núcleo hipotalâmico foi definido como eficiente na estimativa da densidade neuronal e do número total de neurônios hipotalâmicos para posterior comparação entre ratos Wistar não tratados e aqueles tratados com imunossupressores, TAC e MMF. No entanto, devido à distribuição neuronal anisotrópica do hipotálamo, não foi possível obter a densidade volumétrica de cada núcleo hipotalâmico associada à regulação da ingestão alimentar.

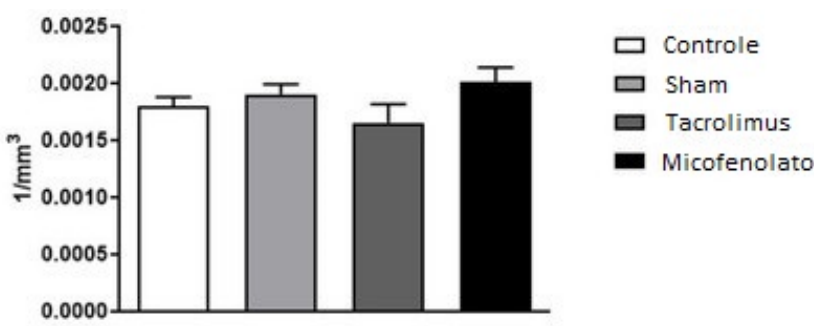

Figura 5 - Morfo-quantificação dos neurônios ventromediais, ANOVA de uma via. Média \pm erro da média. $\mathrm{N}=$ 6 / grupo.

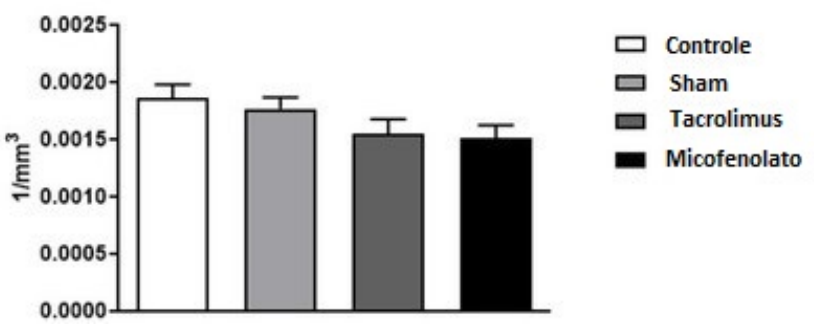

Figura 6 - Morfo-quantificação de neurônios arqueados, ANOVA de uma via. Média \pm erro da média. $N=6$ / grupo.
Com o protocolo pré-clínico utilizado, na tentativa de mimetizar o que acontece com humanos pós-transplantados, não foram encontradas diferenças quantitativas (densidade neuronal) ou qualitativas (morfologia celular) nos núcleos hipotalâmicos estudados. Surpreendentemente, nenhum protocolo de pesquisa semelhante foi encontrado na literatura para analisar os efeitos dessa terapia imunossupressora (TAC e MMF) no hipotálamo de ratos Wistar e sua relação com a obesidade. No entanto, alguns autores investigaram as alterações causadas pela obesidade nos modelos injetados de glutamato monossódico devido à neurotoxicidade celular no hipotálamo 9,11.

Além disso, existem poucos relatos sobre o caráter neurotóxico dos imunossupressores no hipotálamo. Assim, a abordagem quanto ao TAC e MMF como fatores etiológicos relacionados à obesidade é mínima ${ }^{27}$. As correlações diretas entre imunossupressores, núcleos hipotalâmicos e obesidade ainda não são claras, conforme descrito na literatura ${ }^{1}$, as quais referem que o uso de imunossupressores como fator de risco para excesso de peso pós-transplante ainda é controverso.

Por outro lado, vários estudos relatam ganho de peso significativo em pacientes pós-transplante. Muitas vezes, esse ganho de peso pode ser excessivo, resultando em obesidade acompanhada por suas comorbidades associadas. Essa situação é um possível problema de saúde pública crescente e relacionado aos pacientes submetidos a transplantes de rim, fígado e coração ${ }^{1,14}$.

Nenhum ganho de peso acentuado foi identificado nos grupos independentes tratados com TAC e MMF. Portanto, não foi possível verificar aumento de peso significativo nos animais tratados com MMF quando comparados aos grupos Controle e Sham. Provavelmente, essa variação no ganho de peso é explicada principalmente pela ausência de um grupo de medicamentos associados (TAC + MMF) e secundariamente pelo curto período de tratamento.

Em modelo de transplante de membros posteriores, não houve ganho de peso com o uso associado de TAC com triptólido, por via intraperitoneal, por 150 dias em animais transplantados experimentais ${ }^{10}$. Isso pode ser explicado pela inibição do triptólido do ganho de peso induzido pelo TAC. Porém, esse protocolo de tratamento foi realizado por um período prolongado, em comparação com o presente estudo, em 52 dias de diferença, o que pode ser crucial, considerandose a vida útil do rato Wistar.

Da mesma forma, diversos estudos têm demonstrado que a interação medicamentosa combinada pode ser responsável pelo ganho de peso de pacientes pós-transplante, o que comumente ocorre na prática clínica ${ }^{12,17,18,22}$. Alguns autores relataram ganho de peso excessivo, incluindo obesidade em $15 \%$ dos pacientes após transplante de fígado, tratado com associação de imunossupressores TAC e ciclosporina ${ }^{4,6}$. Por outro lado, com TAC e ciclosporina administrados individualmente não foi observado ganho de peso significativo ${ }^{6}$. Da mesma forma, os indivíduos transplantados tratados com ciclosporina no final do primeiro ano adquiriram maior risco de obesidade do que aqueles tratados com TAC no mesmo período ${ }^{8}$. Portanto, alguns estudos corroboram a ausência de ganho de peso significativo em animais tratados apenas com TAC.

O método estereológico utilizado para o estudo morfo-quantitativo dos corpos celulares dos neurônios dos núcleos hipotalâmicos mostrou-se eficiente na aquisição da densidade neuronal e do número total de neurônios hipotalâmicos para posterior comparação entre ratos Wistar não tratados e tratados com imunossupressores, TAC e MMF. No entanto, devido à distribuição neuronal anisotrópica do hipotálamo, não foi possível obter a estimativa volumétrica de cada núcleo hipotalâmico envolvido na regulação da ingestão alimentar. 
O tratamento com imunossupressores TAC e MMF não foi responsável por uma mudança significativa na densidade neuronal nos núcleos hipotalâmicos, PA, LH, DM, VM e ARC neste estudo. Esse dado pode ser explicado, possivelmente, pela ausência de um grupo específico tratado, simultaneamente com TAC e MMF como convencionalmente ocorre nas terapias imunossupressoras de pacientes pós-transplante na clínica.

A ausência de alterações morfo-quantitativas nos neurônios hipotalâmicos nos diferentes grupos estudados não confirma a ausência de efeitos imunossupressores. Assim, métodos complementares são necessários para avaliar os efeitos neurotóxicos dos imunossupressores nessas regiões do cérebro. De acordo com dados da literatura ${ }^{22}$, existe uma possível perda das vias aferentes e eferentes entre o fígado e o hipotálamo durante a cirurgia de transplante de fígado, levando a um distúrbio do papel do fígado na homeostase metabólica, o que pode retardar a saciedade pós-prandial e, portanto, pode influenciar diretamente o consumo excessivo de alimentos. No entanto, nenhum dado conclusivo foi estabelecido para determinar se esse distúrbio tem um efeito direto na ingestão de alimentos e na massa corporal em pacientes pós-transplante. Esses dados apontam para alterações diferentes das investigadas neste estudo, indicando que as alterações na densidade e morfologia neuronal hipotalâmica podem não estar diretamente relacionadas ao ganho de peso encontrado nos pacientes. Além disso, os animais do presente estudo não foram submetidos a nenhuma intervenção cirúrgica semelhante ao procedimento de transplante de fígado, portanto, mais estudos são necessários para investigar a densidade e morfologia neuronal em um modelo animal pós-transplante.

Não há consenso sobre estudos que investiguem os efeitos dos imunossupressores no hipotálamo, mais especificamente nos núcleos hipotalâmicos estudados, quanto ao seu papel na citoarquitetura nessa área. Porém, na literatura, possíveis alterações nos núcleos hipotalâmicos foram citadas parajustificar a desorganização do controle da fome e saciedade exercida pelos neurônios hipotalâmicos envolvidos no desenvolvimento da obesidade ${ }^{3,20}$.

Além disso, com base em modelos experimentais, por meio da injeção de glutamato monossódico em altas concentrações em camundongos, foi relatado o rápido ganho de peso dos animais devido à alta toxicidade causada nas células dos núcleos hipotalâmicos envolvidos com a ingestão de alimentos ${ }^{24,28}$.

A bula aprovada pela Agência Nacional de Vigilância Sanitária (ANVISA) em 29 de outubro de 2015 do medicamento Cellcept ${ }^{\circledR}$, utilizado no estudo como tratamento com MMF informa que nos exames realizados, entre $3 \%$ e $10 \%$ dos pacientes tiveram vertigens, depressão, convulsões, tremores, neuropatias, alucinações, delirium, entre outros efeitos neurológicos e, além destes, apresentaram ganho de peso classificado como muito comum nos casos de transplante cardíaco e comum quando esses pacientes foram submetidos a transplante renal e hepático. No entanto, também foi relatado que o MMF não tem efeito neurotóxico ${ }^{2,16}$, semelhante ao grupo MMF do presente estudo.

Além disso, devido aos princípios dos $3 \mathrm{Rs}^{15}$, havia algumas limitações quanto à especificidade em causar neurotoxicidade $e$ comprometimento direto dos núcleos hipotalâmicos relacionados ao controle da ingestão alimentar e, portanto, desencadeamento da obesidade. Porém, o uso sequencial de animais neste estudo não invalida os resultados obtidos no modelo experimental desenvolvido com animais tratados com TAC e MMF.

Sintetizando, a interação medicamentosa entre imunossupressores e outras drogas pode ser responsável pelo quadro neurotóxico de certas regiões do sistema nervoso, favorecendo o ganho de peso e o desenvolvimento da obesidade, porém estudos pré-clínicos futuros são necessários para confirmar essa hipótese.

\section{CONCLUSÃO}

Os tratamentos com imunossupressores TAC e MMF isolados não foram capazes de modificar os aspectos morfológicos e de densidade celular dos núcleos hipotalâmicos durante o período de suplementação. Além disso, não foi observada obesidade com o protocolo de administração de imunossupressor utilizado.

\section{REFERÊNCIAS}

1. Anastácio LR, Pereira MC de A, Vilela EG, Lima AS, Correia MITD. Overweight in liver transplant recipients Excesso de peso em pacientes submetidos ao transplante hepático. Rev Col Bras Cir. 2013;40(6):502-6. doi: 10.1590/s0100-69912013000600014.

2. Anghel D, Tanasescu R, Campeanu A, Lupescu I, Podda G, Bajenaru O. Neurotoxicity of immunosuppressive therapies in organ transplantation. Maedica (Bucur).2013;8(2):170-5.PMID:24371481; PMCID: PMC3865126.

3. Berthoud HR. The neurobiology of food intake in an obesogenic environment. Proc Nutr Soc. 2013;71(4):478-87. doi: 10.1017/ S0029665112000602

4. Bianchi G, Marchesini G, Marzocchi R, Pinna AD, Zoli M. Metabolic Syndrome in Liver Transplantation : Relation to Etiology and Immunosuppression. 2008;1648-54. doi: 10.1002/lt.21588.

5. Braun F, Teren K, Wilms P, Günther R, Allmann J, Broering DC, Küchler T. Quality of Life After Liver Transplantation. Transplant Proc [Internet]. 2009;41(6):2564-6. http://dx.doi.org/10.1016/j. transproceed.2009.06.030.

6. CanzanelloVJ, SchwartzL, TalerSJ, TextorSC, WiesnerRH, PoraykoMK, Krom RAF. Evolution of cardiovascular risk after liver transplantation: A comparison of cyclosporine A and tacrolimus (FK506). Liver Transplant Surg. 1997;3(1):1-9. doi: 10.1002/lt.500030101.

7. Charlton M, Rinella M, Patel D, McCague K, Heimbach J, Watt K. Everolimus Is Associated with Less Weight Gain Than Tacrolimus 2 Years after Liver Transplantation: Results of a Randomized Multicenter Study. Transplantation. 2017;101(12):2873-82. doi: 10.1097/TP.0000000000001913.

8. Everhart JE, Lombardero M, Lake JR, Wiesner RH, Zetterman RK, Hoofnagle JH. Weightchange and obesity after liver transplantation: incidenceand risk factors. Liver Transpl Surg [Internet]. 1998;4(4):28596. doi: 10.1002/lt.500040402.

9. Gomes JR, Freitas JR, Grassiolli S. Effects of Physical Exercise on the Intestinal Mucosa of Rats Submitted to a Hypothalamic Obesity Condition. Anat Rec.2016;299(10):1389-96. doi:10.1002/ar.23453.

10. Gu C, Liu F, Luo X, Zhou X, Yang J, Levin LS. Triptolide Reduces the Required Dose of Tacrolimus by Attenuating Inflammation, Enhancing Immunosuppression, and Increasing Donor Chimerism in a Heterotopic Hindlimb Transplantation Model. Plast Reconstr Surg. 2016;138(6):1243-53. doi: 10.1097/PRS.0000000000002770.

11. Jin YJ, Cao PJ, Bian WH, Li ME, Zhou R, Zhang LY, Yang MZ. BDNF levels in adipose tissue and hypothalamus were reduced in mice with MSG-induced obesity. Nutr Neurosci. 2015;18(8):376-82. doi: 10.1179/1476830515Y.0000000039.

12. Kalluri HV, Hardinger KL. Current state of renal transplant immunosuppression: Present and future. World J Transplant. 2012;2(4):51. doi: 10.5500/wjt.v2.i4.51.

13. Kugler C, Einhorn I, Gottlieb J, Warnecke G, Schwarz A, Barg-Hock H, Bara $\mathrm{C}$, Haller $\mathrm{H}$, Haverich A. Postoperative weight gain during the first year after kidney, liver, heart, and lung transplant: A prospective study. Prog Transplant. 2015;25(1):49-55. doi: 10.7182/pit2015668.

14. Lopez-Vilella R, Sánchez-LázarolJ, Martínez-Dolz L, Almenar-Bonet $\mathrm{L}$, Marqués-Sulé E, Melero-Ferrer J, Portolés-Sanz M, RiveraOtero M, Domingo-Valero D, Montero-Argudo A. Incidence of Development of Obesity After Heart Transplantation According to the Calcineurin Inhibitor. Transplant Proc. 2015;47(1):127-9. http://dx.doi.org/10.1016/j.transproceed.2014.11.025. 
15. Ministério da Ciência T e I, CONCEA CN de C de EA-. Diretriz brasileira para o cuidado e a utilização de animais para fins científicos e didáticos. Diário Oficial da União - 25/09/2013. 2013;51.

16. Mukherjee S, Mukherjee U. A Comprehensive Review of Immunosuppression Used for Liver Transplantation. J Transplant [Internet]. 2009;2009:1-20. doi: 10.1155/2009/701464.

17. Neal DAJ, Gimson AES, Gibbs P, Alexander GJM. Beneficial effects of converting liver transplant recipients from cyclosporine to tacrolimus on blood pressure, serum lipids, and weight. Liver Transplant. 2001;7(6):533-9. doi: 10.1053/jlts.2001.24637.

18. Palmer M, Schaffner F, Thung SN. Excessive Weight Gain After Liver Transplantation. Transplantation. 1991;51(4):797-800. doi: 10.1097/00007890-199104000-00012.

19. Paxinos G, Watson C. The Rat Brain in Stereotaxic Coordinates (1997). Acad Press. New York. 5a edição, 2005.

20. Plagemann A, Harder $T$, Rake A, Voits M, Fink $H$, Rohde W, Dörner $G$. Perinatal elevation of hypothalamic insulin, acquired malformation of hypothalamic galaninergic neurons, and syndrome X-like alterations in adulthood of neonatally overfed rats. Brain Res. 1999;836(1-2):146-55. doi: 10.1016/s00068993(99)01662-5.

21. Ribeiro $H$ de $S$, Anastácio LR, Ferreira LG, Lagares ÉB, Lima AS, Correia MITD. Prevalence and factors associated with dyslipidemia after liver transplantation. Rev Assoc Med Bras. 2014;60(4):365-72. doi: 10.1590/1806-9282.60.04.016.
22. Richards J, Gunson B, Johnson J, Neuberger J. Weight gain and obesity after liver transplantation. Transpl Int. 2005;18(4):461-6. doi: 10.1111/j.1432-2277.2004.00067.x.

23. Richardson RA, Garden OJ, Davidson HI. Reduction in energy expenditureafter liver transplantation. Nutrition.2001;17(7-8):585-9. doi: 10.1016/s0899-9007(01)00571-8.

24. Rojas-Castañeda JC, Vigueras-Villaseñor RM, Chávez-Saldaña M, Rojas P, Gutiérrez-Pérez O, Rojas C, Arteaga-Silva M. Neonatal exposure to monosodium glutamate induces morphological alterations in suprachiasmatic nucleus of adult rat. Int J Exp Pathol. 2016;97(1):18-26. doi: 10.1111/iep.12157.

25. Torterolli F, Watanabe RK, Tabushi Fl, Peixoto IL, Afonso P, Nassif N, Tefilli NL, Rocha SL, Malafaia O. BAR, SOFT and DRI post-hepatic transplantation: what is the best for survival analysis? Arq Bras Cir Dig. 2021;34(1):e1576. doi: 10.1590/0102-672020210001e1576.

26. Tran M, Foster CE, Kalantar-zadeh K, Ichii H. Kidney transplantation in obese patients. World J Transplant ${ }^{\odot}$ 2016. 2016;6(1):135-44. doi: 10.5500/wjt.v6.i1.135.

27. Watanabe ALC, Matias JEF. Does the association of tacrolimus and mycophenolate mofetil change the healing of the abdominal wall? Study in rats submitted to ischemia and kidney reperfusion. Arq Bras Cir Dig. 2020;33(4):1-5. doi: 10.1590/0102-672020200004e1551.

28. Železná B, Maixnerová J, Matyšková R, Haugvicová R, Blokešová D, Maletínská L. Anorexigenic effect of cholecystokinin is lost but that of cart (cocaine and amphetamine regulated transcript) peptide is preserved in monosodium glutamate obese mice. Physiol Res. 2009;58(5):717-23. doi: 10.33549/physiolres.931511. 